\title{
Education interculturelle et éducation à la citoyenneté: Un détour par la sociologie de la nation
}

\section{Françoise Lorcerie}

La question du rapport entre éducation interculturelle et éducation à la citoyenneté gagne à être replacée dans le contexte socio-politique où elle se pose aujourd'hui dans les pays occidentaux. Ce contexte se caractérise par une crise, voire plus rarement une récusation de l'Etat-nation "classique», qui reposait sur une opposition ethnique naturalisée entre established et outsiders combinée avec un principe démocratique. On observe une saillance sociale nouvelle de la pluralité culturelle comme problème appelant un traitement public et du pluralisme comme valeur. L'éducation publique est un des sites de cette crise, en même temps qu'un des espaces où son traitement est envisageable. Ainsi, au Québec, l'emblème de l'interculturel fut expérimenté dans la cadre de la réelaboration symbolique de la nation québécoise (depuis la fin des années 1970), avant d'être quelque peu mis en retrait par les pouvoirs publics au bénéfice d'autres emblèmes plus explicitement inclusionnaires. En France, la revitalisation récente de l'éducation à la citoyenneté et la reconnaissance (timide) des différences culturelles s'expliquent également dans le contexte de ce qu'on peut appeler la crise de l'intégration assimilationniste et l'inflexion qui se fait jour du "régime de tolérance» des différences.

Difficile question que celle de l'apport d'une perspective interculturelle à la citoyenneté et, a fortiori, à l'éducation à la citoyenneté. En effet, aucun des concepts que mobilise cette question ne peut être considéré comme stabilisé.

Le concept de citoyenneté, notamment, qui sert de référence dans la question, est au cœur des débats que développe aujourd'hui la philosophie politique.

A quoi pourrait ressembler une conception de la citoyenneté appropriée étant donné le contexte des sociétés pluralistes modernes? Quels sont les devoirs et les pratiques autour desquels une telle conception serait construite?

Daniel Weinstock termine sur ces questions étrangement ouvertes un état des lieux des «réponses» fournies par la citoyenneté au problème du pluralisme des sociétés modernes, du point de vue de la philosophie politique (Weinstock, 1998, p. 92). 
Au point que le concept de citoyenneté lui parait appeler désormais une étude autonome. Ce scepticisme pourrait être contesté, certes. Mais le constat est argumenté.

Distinguant le pluralisme des valeurs (dit axiologique) et le pluralisme culturel, Weinstock montre que dans l'un et l'autre cas, les solutions proposées jusqu'ici par John Rawls et ses discutants (libéraux ou communautariens), ou par Habermas et les tenants de la démocratie délibérative (dans l'une ou l'autre de ses versions) ne sont pas satisfaisantes:

L'absence de consensus normatif spontané face à laquelle nous place le pluralisme axiologique fait de sorte que nous nous devons de regarder du côté des délibérations démocratiques entre citoyens afin de créer de tels consensus. (Weinstock, 1998, p. 81).

Or cette solution se heurte à de très nombreux aléas. Quant à «l'absence d'identité partagée que nous impose le pluralisme culturel, (elle) entrâne la nécessité, pour l'Etat soucieux d'assurer la viabilité de ses institutions, de promouvoir l'identité de citoyen qui peut au moins en principe être partagée par tous les membres de la société, indépendamment de leurs identités culturelles» (Weinstock, 1998, p. 81). Mais en quoi consiste-t-elle?

Prenant acte du fait que le chantier conceptuel est ouvert et loin d'être clos, c'est sur le chantier lui-même que nous voudrions centrer notre questionnement. En quoi l'incertitude sur la nature de la citoyenneté et le renouveau d'intérêt pour la notion sont-ils liés au changement des sociétés nationales, à la saillance nouvelle de leur pluralisme axiologique et culturel? En quoi la réforme de l'éducation à la citoyenneté répond-elle à ce changement global, et quelle part laisset-elle à l'éducation interculturelle? Pour prendre l'exemple de la France, une éducation civique rénovée s'applique au collège depuis quelques années (Audigier $\&$ Lagelée, 1996), et un enseignement nouveau vient d'être introduit au lycée, déclinant la citoyenneté sous une variété de problématiques sociales, juridiques et politiques. La dimension interculturelle n'y figure pas, mais le programme mentionne «la problématique de l'unité politique et du respect des différences légitimes». La mention et surtout la thématisation des différences et particularismes culturels est nouvelle dans le discours officiel de l'Education nationale française. Avant, on n'en parlait pas sinon pour affirmer qu'elles étaient respectées. Pourquoi ce changement et quelle est sa consistance?

Prendre la question par le biais des sciences sociales amène à conduire l'analyse dans le cadre du paradigme de l'ethnicité, un paradigme dont les grandes lignes furent tracées par Max Weber dans un texte célèbre (Weber, 1956). Il combine de façon complexe des dimensions psychologiques, sociologiques et politiques, autant dire qu'il défie les frontières académiques habituelles. Au centre de l'interrogation, les concepts de nation et d'identité 1 .

Nous traiterons d'abord de la nature ethnique de la nation, d'après Weber et Mauss, prolongés par Elias. Dans l'Etat-nation classique, principe ethnique et principe démocratique forment un attelage intimement uni, sinon stable. L'école 
y contribue tant par son curriculum que par ses fonctionnements. L'idée de valoriser la pluralité culturelle de la société, qui est comprise dans l'éducation interculturelle, n'a pas de place dans cette configuration. Elle peut en acquérir c'est ce que nous examinerons en seconde partie - quand cette configuration entre en crise ou devient inadéquate du fait du changement social, comme on le voit sur l'exemple du Québec ou de la France depuis les années 1980. Nous sommes à une époque et dans un espace où, l'ethnonationalisme venant en contradiction avec la visée démocratique de l'Etat-nation, c'est celle-ci qui l'emporte et tend à remodeler l'idéal de la nation. Dans ce contexte, l'école est sollicitée par le politique pour contribuer à la transformation des mentalités et des pratiques civiles, et l'éducation à la citoyenneté est remise en chantier.

\section{Established et outsiders: L'ethnicité dans l'Etat-nation}

\section{Les fondements théoriques}

Le paradigme de l'ethnicité, tel que fondé par Weber, a pour énigme centrale la question de la production sociale d'une certaine forme d'identité communautaire que l'on peut appeler une identité tribale au cœur des sociétés modernes, l'identité d'individus qui croient qu'ils partagent (ou que d'autres partagent) une communauté d'origine. Weber part de la vision essentialiste de la parenté ethnique, qu'il trouve dans l'ethnologie et la sociologie de son temps, pour s'en écarter radicalement par trois recadrages. Premièrement, l'appartenance ethnique n'est pas une question d'essence, pas une question d'être-en commun (le concept d'ethnie est "un fourre-tout inutilisable», écrit-il), mais une question de croyance, de représentation sociale. Deuxièmement, cette croyance est largement induite par certaines dynamiques sociales, notamment par l'organisation et la vie politiques nationales. C'est au sein de ces dynamiques que les schèmes d'imputation des différences prennent consistance et que le contenu des ressemblances tribales s'élabore, symboliquement et matériellement, et, précise-t-il, qu'elles se chargent plus ou moins de pathos, de passion. L'ethnicité est tout autant sinon plus, selon Weber, caractéristique des groupes dotés d'une organisation politique, qu'un trait de groupes minoritaires. Troisièmement, cette croyance se déploie toujours à l'encontre d'autres, tenus pour essentiellement différents et infériorisés à raison de cette différence, tandis que l'on se rehausse collectivement.

Le sentiment national n'est ainsi rien d'autre, d'un point de vue sociologique, qu'une forme d'identité ethnique. A la même époque (et sans connexion), Marcel Mauss avance une idée voisine dans son texte inachevé sur la nation, et il approfondit la question du rapport entre cette identité et l'activité politique (Mauss, 1969). La nation est pour lui caractérisée par une «coïncidence» entre pouvoir "démocratique à quelque degré», unité linguistique et unité ethno-culturelle. Mais, note-t-il, la coïncidence ne résulte pas d'une transmission, c'est 
une construction sociale. Les traits qui apparentent la nation à l'ethnos sont, dans la nation, pour partie au moins, l'effet du processus "d'individuation» qui oppose et lie tout à la fois la nation-Etat à son environnement. Mauss voit la nation moderne en auteur de son identité ethnique, laquelle est générée dans le conflit ou dans le concert des autres nations. La nation moderne se prévaut d'une «race», d'une langue nationale, d'une esthétique nationale, d'un "caractère national», qui sont en réalité ses créations, en même temps qu'elle se prévaut d'une volonté propre, d'une idée du progrès, et qu'elle se dote d'un droit public, d'un marché intérieur unifié, et d'institutions démocratiques reposant sur la "corrélation» entre l'idée de patrie et celle de citoyen: "Une nation digne de ce nom a sa civilisation, esthétique, morale et matérielle, et presque toujours sa langue. Elle a sa mentalité, $s a$ sensibilité, $s a$ moralité, $s a$ volonté, $s a$ forme de progrès, et tous les citoyens qui la composent participent en somme à l'Idée qui la mène» (p. 591; souligné par l'auteur).

La réflexion de Mauss, reprise et actualisée aujourd'hui (Babibar, 1988; Lorcerie, 2002), amène à scruter le couplage qui s'effectue entre ethnos et dèmos, entre le peuple ethnique et le peuple souverain, dans l'Etat-nation. Couplage? hybridation? Ces mots sont des métaphores et non des concepts. Le trait d'union qui lie Etat et nation a été aussi peu étudié en sociologie politique qu'en philosophie politique. Raymond Aron propose en 1961 cette définition de travail: «Le type idéal d'un Etat national est celui d'une unité politique, dont les citoyens appartiennent tous à une même culture et manifestent la volonté de vivre en une communauté autonome» (cité par Tenzer, 1994, p. 469). Autrement dit, l'Etatnation se caractérise typiquement par une unité double, juridico-institutionnelle et culturelle, et par une cohésion subjective qui fonde ou perpétue la séparation de l'unité en question d'avec son environnement.

Ce caractère hybride s'exprime historiquement à travers la malléabilité de l'acception pratique du concept de citoyenneté dans la vie politique (Colas, 2000), par son "profond dualisme» (Smith, 1991), indépendamment les doctrines officielles de la nation et de la citoyenneté (on sait qu'en France, notamment, la doctrine de la citoyenneté nationale insiste sur le côté politique du lien). C'est ce que traduisent, si l'on y prend garde, les dimensions hétérogènes habituellement associées au concept de citoyenneté. Les auteurs qui tentent de mettre à plat le contenu de la citoyenneté lui attribuent toujours au minimum: une dimension juridique (le fait d'être sujet de droits), une dimension politique (la participation politique), et une dimension communautaire (le fait d'être «membre reconnu d'une collectivité, le plus souvent identifiée à la Nation", écrivent Barrère et Martuccelli (1998); ou «le degré d'identification à l'identité collective qui caractérise la société globale», selon Pagé, 2001).

Appelons ethnonationalisme la postulation ethnique de l'Etat national. En raison du caractère hybride de l'attelage institutionnel que constitue l'Etat-nation, l'ethnonationalisme est, par hypothèse, toujours en rapport d'association (de coexistence et de tension) avec (1) le principe individuo-universaliste de l'Etat na- 
tional. Par ailleurs, en raison de la différenciation possible des identifications ethniques au sein de la communauté nationale, l'ethnonationalisme est aussi toujours en rapport d'association (de coexistence et de tension) avec (2) les ethnicités minoritaires éventuellement présentes. Des rapports qu'il faut penser, d'un point de vue sociologique, comme des dynamiques mobilisant des acteurs et s'articulant en luttes sociales.

A ce point, la sociologie politique de la citoyenneté trouve à s'appuyer sur la psychologie sociale des relations entre groupes de statuts inégaux, et sur la sociologie de la domination symbolique. Sans détailler, mentionnons l'intérêt pour notre propos de l'hypothèse de la "configuration establishedloutsiders", due à Norbert Elias. Elle peut se formuler ainsi: tout groupe humain établi sur un territoire, placé au contact de nouveaux venus, engage au minimum une lutte symbolique par laquelle il dénigre les intrus et exalte son propre charisme collectif, de sorte à tenir les outsiders éloignés du pouvoir. L'idéologie par laquelle le groupe symbolise sa différence et sa supériorité est secondaire. Ce qui importe est que cette idéologie soit capable d'alimenter sa fierté collective, le "charisme de groupe», et de renforcer sa cohésion à l'encontre des intrus, de sorte à entretenir le monopole qu'il détient sur les positions de pouvoir. Cette hypothèse théorique n'est pas spécifique aux dynamiques communautaires dans l'Etat-nation, elle est développée par Elias notamment à l'occasion d'une étude des problèmes de sécurité urbaine d'une petite ville britannique d'ancienne population ouvrière (Elias \& Scotson, 1964/1997). Mais, selon Elias lui-même, elle s'applique "de façon exemplaire» aux logiques d'identification dans la nation.

Par hypothèse, la relation au temps et au territoire jouent un rôle décisif dans la genèse du "charisme de groupe» dans la relation établis/intrus. Par suite, la domination symbolique s'ethnicise normalement au sein des collectivités nationales, en même temps que sont ethnicisés, corrélativement, les individus collectivement rejetés comme intrus (en tant qu'étrangers, ou en tant qu'immigrés). Des conflits s'ensuivent portant sur la façon dont les individus se définissent et sont définis personnellement et collectivement, - sachant que, «dans tous les cas, les nouveaux venus s'appliquent à améliorer leur position, et les groupes établis à maintenir la leur» (Elias \& Scotson, 1964/1997, p. 340). Ces conflits sous-tendent le développement des «crises de l'intégration», dont nous parlerons plus loin.

\section{L'école et l'entretien de l'ethnicité nationale}

S'il est vrai que l'école assure la reproduction de la société (c'est une idée qui n'est pas née avec Bourdieu), on peut faire l'hypothèse qu'elle répercute l'hybridité constitutive de l'Etat-nation, selon des modalités variables puisque ce dualisme est socialement instable: d'une façon ou d'une autre, elle concourt à l'entretien de l'ethnicité nationale en même temps qu'elle forme le peuple souverain.

La propagation de l'idéologie nationale est une des voies possibles. Mauss y insiste. Pour lui, l'école nationale a pour rôle «l'accentuation» des "caractères col- 
lectifs de la nation», - contre les identités minoritaires, donc, mais aussi en relativisant la visée civique et morale sur laquelle insistent pourtant les premiers manuels républicains en France (Déloye, 1994). Dans l'école française, la modalité "patriote» de l'ethnicité nationale fut saillante aux lendemains de l'installation de la Troisième République, qui faisait suite à la défaite de 1870 devant la Prusse. Mais elle s'estompa dès l'entre-deux-guerres, avec le pacifisme qui découla de la Première guerre mondiale (Loubes, 2001). Une autre modalité, sur laquelle Suzanne Citron notamment a attiré l'attention et qui dura jusqu'aux années soixante au moins, est la transformation - à l'école primaire surtout - de l'histoire de la France en «histoire de France», geste collectif sacralisant la France éternelle, victorieuse de l'Ancien régime et mère des droits de l'homme (Citron, 1989; Nora, 1997).

On peut s'attendre par ailleurs à ce que les fonctionnements ordinaires (sociaux) de l'école reproduisent la domination ethnique qui prévaut dans la société. C'est ce qui a été mis en évidence récemment dans le cas de l'école française. L'enquête a montré que le couple ethnique Français/immigrés est présent dans les fonctionnements ordinaires de l'école urbaine: dans la genèse et le traitement de la violence scolaire, dans les relations établies par les agents scolaires avec les parents immigrés, dans l'émergence et le traitement des «affaires de foulards», dans les dynamiques de ségrégation entre établissements et en leur sein (Barthon, 1998; Debarbieux, 1996; Debarbieux, Garnier, Montoya \& Tichit 1999; Lorcerie, 1996; Payet, 1995, 1997). Cette modalité de l'ethnonationalisme à l'école n'est pas du même ordre que les modalités relevant du curriculum. Il ne s'agit pas de fonctionnements prescrits par la hiérarchie administrative. Ce sont des fonctionnements "par le bas» et systémiques, pas forcément délibérés. Les agents scolaires et les parents appartenant aux groupes majoritaires (dans la structure de domination nationale) en sont les protagonistes d'un côté, les jeunes issus de l'immigration minorisée (extra-communautaire) et leurs parents, de l'autre. Les uns et les autres ne font qu'appliquer aux interactions et enjeux scolaires les schèmes ethnicisés qui prévalent dans l'environnement social (De Rudder, Poiret \& Vourc'h, 2000). Cette découverte, jointe à d'autres analogues dans les sphères du travail, du logement, des loisirs, a ouvert la voie à l'attestation publique de l'existence des «discriminations ethniques et raciales» en France (Haut conseil de l'intégration, 1998; Lorcerie, 2000), suscitant en réponse des politiques publiques nouvelles.

Les développements précédents amènent à interroger la construction de la discrimination ethnique comme défi politique. En effet, si l'on admet que l'opposition established vs outsiders est constitutive de l'identification nationale dans l'Etat-nation classique, on ne peut pas penser que les fonctionnements discriminatoires soient nouveaux socialement (Noiriel, 1988). Or aujourd'hui ils tendent à être perçus comme moralement inacceptables et dangereux pour la cohésion nationale. Pourquoi? L'écart paraît s'être creusé entre l'ethnonationalisme et le principe individuo-universaliste qui étaient classiquement mêlées dans l'Etat-nation d'avant. 


\section{Crise de l'Etat-nation et changement scolaire: la revitalisation de l'éducation à la citoyenneté}

\section{L'hypothèse d'une crise contemporaine de l'Etat-nation}

Les modalités scolaires de l'ethnicité aujourd'hui s'expliquent en partie au moins par des facteurs qui n'ont rien à voir avec la crise de la forme stato-nationale: par la crise urbaine et la polarisation sociale croissante des quartiers, et par la «massification» de l'enseignement secondaire au cours des années 1980-1990 (Van Zanten, 2001). Cependant, dans un système causal complexe, deux facteurs généraux se laissent distinguer, qui ont directement déterminé le renouvellement (qui s'esquisse) des politiques publiques en matière d'intégration des immigrés et de solidarité nationale, à savoir: la crise de l'intégration nationale, et l'émergence de nouvelles attentes de justice démocratique.

La question de la crise de l'intégration nationale a été soulevée en France au tournant des années 1990, tandis que battait son plein la polémique sur l'assimilabilité des immigrés (Schnapper, 1991). Crise, le diagnostic général n'est guère contesté et les soubresauts de la vie politique nationale autour de la place et du traitement des immigrés durant vingt-cinq ans (du milieu des années 1970 à la fin des années 1990) ne le démentent pas. Pour Dominique Schnapper, la crise est essentiellement due à l'altération des grandes institutions qui assuraient auparavant la formation de la cohésion nationale: l'école, mais aussi l'armée, les syndicats, et même l'Eglise. Cette crise, les immigrés - leurs cultures, leurs conduites - en sont moins la cause qu'ils ne la révèlent, pose la sociologue.

Cette analyse écarte donc, à juste titre, l'explication anti-sociologique de la crise par les différences culturelles objectives des immigrés. Mais on peut lui objecter qu'elle ne prend pas en charge le facteur particulier qu'est l'intensité de la catégorisation sociale des «immigrés» comme intrus. Poursuivons en effet le raisonnement dans la ligne que suggère la psychologie sociale: si les immigrés manifestent la crise sans la produire ou sans que leurs cultures fassent obstacle à leur intégration, alors c'est que leur visibilité est en cause aussi dans la crise, autrement dit l'imputation d'altérité dont ils sont l'objet dans la catégorisation sociale dominante. Le stéréotypage récurrent de l'immigré (ainsi désigné dans son statut d'intrus de génération en génération) sape l'imaginaire de l'unité nationale, il amène le majoritaire au fantasme de la fragmentation sociale (Wieviorka, 1996), et il induit des pratiques discriminatoires, lesquelles à leur tour suscitent une variété de réactions de la part de ceux qui les subissent. Ainsi, les revendications de citoyenneté et de dignité dont les élites immigrées sont porteuses, les luttes qui s'organisent à partir du milieu associatif contre les discriminations, procèdentelles de la crise de l'intégration nationale en même temps qu'elles y participent. De même (à un autre point de vue) que les déviances et l'anomie développées par les jeunes issus de l'immigration dans les zones urbaines de «relégation». 
La force du stéréotype s'enracine dans le traumatisme colonial, dont l'imaginaire est entretenu plus ou moins fort de part et d'autre (Stora, 1999), mais elle se perpétue au travers de l'activité sociale des acteurs majoritaires et minoritaires, ici et maintenant, qu'il s'agisse de communication publique, de mobilisations collectives ou de simples échecs interactionnels.

\section{Le renouvellement des normes démocratiques}

Le renouvellement des normes démocratiques aujourd'hui est un autre facteur de la crise de l'Etat-nation classique, d'autant que l'Union européenne s'en fait le promoteur, ainsi que le Conseil de l'Europe. Interagissant avec la crise de l'intégration nationale, le changement normatif modèle un nouvel imaginaire de la cité politique ${ }^{2}$. Pour le dire sommairement, les philosophies contemporaines de la démocratie dénoncent l'oppression des minorités par les majorités nationales; le pluralisme axiologique et culturel est au centre de leurs débats sur la nature de la démocratie.

Si la société est fondamentalement plurielle (c'est l'axiome commun de ces philosophies), alors la solidarité nationale - pièce maîtresse de l'architecture des institutions démocratiques - ne saurait se fonder sur la ressemblance culturelle (réelle ou supposée) ni sur la ressemblance morale (le partage d'un même corpus de valeurs), mais sur autre chose, qu'il faut élucider. La réponse, les philosophes de la démocratie la fondent, par delà la diversité de leurs systèmes, sur une anthropologie de l'intersubjectivité. A partir de quoi ils vont chercher quelles dispositions institutionnelles sont propres à garantir la réciprocité intersubjective, le pluralisme étant pris comme un fait. Rawls s'attache à spécifier les règles constitutionnelles qui rendent possible et stabilisent la coopération sociale dans l'équité, Habermas insiste quant à lui sur l'aspect processuel de l'accord, dans une société démocratique ouverte, «aux frontières poreuses» (Habermas, 1996; Rawls, 1993; Touraine, 1997).

Dans tous les cas, le politique est nettement distingué du culturel (et non plus associé à la culture majoritaire comme dans l'attelage ethno-démocratique qui sous-tend l'Etat national classique). Le politique démocratiquement organisé garantit aux membres de la société la jouissance de leurs droits fondamentaux, parmi lesquels le droit à être eux-mêmes et le droit à entretenir des conceptions opposées du bien (pour reprendre à Rawls son vocabulaire). Distinction du politique et du culturel, donc, mais responsabilité du politique - là est le point. Il se peut que des matières culturelles ou identitaires requièrent l'intervention du politique. Moins pour interdire que pour protéger, spécialement les groupes minoritaires.

C'est à ce titre que certains, comme Michael Taylor, réclament l'institutionnalisation du droit à l'identité culturelle: «La reconnaissance est un besoin humain vital» (Taylor, 1992, p. 42), or il n'est pas satisfait pour les minoritaires (c'est-à-dire les groupes minorisés dans la société) sans une intervention expresse de l'Etat pour garantir la survie culturelle du groupe. La position philosophique 
que répercutent, aujourd'hui, les normes européennes serait plus proche de celle d'un Rawls, qui fait du droit à l'identité une condition primitive de la justice des institutions politiques. Il faudra, si l'on suit Rawls et les philosophes dits «libéraux», que l'Etat intervienne non pas pour «reconnaître» directement les identités culturelles présentes dans la société comme le souhaitent Taylor et les philosophes d'inspiration "communautarienne», mais pour tenir sous contrôle les effets sociaux et psycho-sociaux défavorables de la domination du majoritaire (ce qui ne se réduit pas à la lutte contre les discriminations). En la matière, les philosophies modernes de la démocratie demandent au minimum un interventionnisme public de protection de la dignité des identités minoritaires.

\section{Inflexion du "régime de tolérance» et thématique de la pluralité dans l'école}

Le développement de la crise de l'intégration nationale, manifesté par la politisation de la question des immigrés dans une longue crise politique, jointe à l'hégémonie de nouvelles normes politiques qui commencent à être dotées d'une traduction juridique à l'échelle européenne (voir notamment la directive 2000/43 de la commission européenne «relative à la mise en œuvre du principe de l'égalité de traitement entre les personnes sans distinction de race ou d'origine ethnique») ont fini par déboucher sur un début de changement des politiques de l'identité nationale. En France, cela s'est amorcé à la faveur de l'alternance gouvernementale de 1997. Un dispositif de lutte contre les discriminations ethniques a été instauré, un protocole a été conclu pour garantir l'extension au culte musulman du régime des libertés publiques dont bénéficient les autres religions, et le thème de la "diversité» et de la "culture commune» a fait son apparition dans le nouvel enseignement d'éducation à la citoyenneté au lycée (Education civique, juridique et sociale, ECJS). Autant de signes qu'un processus d'inflexion du "régime de tolérance» des différences (Walzer, 1997) est engagé dans la société et se déploie désormais aux plans politique et institutionnel.

Le Québec a connu semblable inflexion de son «régime de tolérance», en plus marqué et en plus rapide, après que, par la loi 101 (1977), il a décidé d'une politique de francisation des immigrants. Destiné à accroître graduellement le poids des francophones dans la province binationale et à garantir la survie de la langue française dans l'Amérique du Nord anglophone, ce choix politique a induit une profonde transformation de l'identité sociale et politique québécoisefrancophone. De socialement minoritaire au Québec (bien que majoritaire en nombre) et a fortiori au Canada, elle est devenue politiquement majoritaire, mais moins ethnique et plus politique, expressément inclusionnaire.

C'est à ce titre que la thématique de l'interculturalisme a été publiquement assumée et élaborée dès 1983 (par emprunt à un vocabulaire employé à l'époque en France). Le mot d'interculturel a été sensiblement mis en retrait ces dernières années en raison des représentations essentialistes qu'il suscite (ou qu'il ne combat pas) chez les destinataires de la politique - malgré l'approfondissement uni- 
versitaire dont il a fait l'objet (Ouellet, 1991; Ouellet \& Pagé, 1991). Mais le gouvernement québécois n'a eu de cesse, durant ces vingt ans, que de chercher la voie d'un universalisme symbolique qui évite aussi bien l'écueil de la réification des cultures des immigrants, que l'écueil de leur déni, tout en mettant l'accent sur le partage d'un espace commun ou d'une culture commune (les experts continuent à disputer ce point). "Québécois de toutes origines» est la désignation officiellement promue aujourd'hui, en remplacement de "Québécois des communautés culturelles», qui reste entachée de présupposés majoritaires (Mc Andrew, 2001, 2002).

On voit sur les exemples de la France et du Québec que les enjeux sociaux et politiques régissent l'interprétation de la thématique de la pluralité culturelle, lorsqu'elle est mise en avant, - enjeux que, pour l'essentiel, l'éducation relaie.

Ainsi pour l'interculturel, terme éminemment polysémique (comment en serait-il autrement quand le concept même de culture n'est fixé ni dans son usage en sciences sociales ni dans le débat philosophique contemporain [(Cuche, 1996; Mesure \& Renaut, 1999], et qu'il se charge si facilement d'affect?). Lorsque les milieux français de l'expertise créent le néologisme interculturel, dans la deuxième moitié des années 1970, et l'implantent au Conseil de l'Europe (Porcher, 1981), leur discours est un discours d'inclusion et de renouvellement de l'unité culturelle de la nation. Mais ce discours vient en contrepoint de la communication publique sur l'immigration et de la politique qui est alors celle du gouvernement français, discours et politique que l'on peut qualifier de réaction nationaliste (incitation au départ, expulsion de jeunes, politique sécuritaire). Dès lors, la thématique de l'interculturel a travaillé à cette époque de façon paradoxale. La mise en ouvre scolaire de la consigne de «valorisation des cultures d'origine» par des "activités interculturelles» a pu tourner à l'essentialisme culturel et à l'exclusion symbolique d'autant plus facilement qu'aucun projet politique inclusionnaire n'était formulé par le politique (Berque, 1985; Lorcerie, 1989; Sayad, 1988).

Aujourd'hui, en France et au Québec - en lien avec l'inflexion des régimes de tolérance évoquée plus haut - l'importance d'une éducation à la citoyenneté est mise en exergue, et celle-ci est conçue comme incluant une réflexion sur la diversité (ethno)culturelle constitutive de la société. Les deux pays ont écarté des programmes officiels - le Québec plus nettement que la France - «la conception nationale unitaire» de la citoyenneté, qui consacre la domination du groupe majoritaire, et ils ont plutôt opté - le Québec plus nettement que la France encore une fois - pour l'enseignement d'une "conception délibérative pluraliste», parfois dite "républicaine», accordant une priorité à la participation, notamment à la délibération. Selon cette conception, inspirée de Habermas, Miller, Kymlicka, ..., «c'est par la délibération active entre citoyens que se fait l'identité nationale dans des dimensions substantives» (Pagé, 2001, p. 47). Le Conseil supérieur de l'éducation du Québec, organe consultatif, a récemment donné de l'éducation à la citoyenneté un exposé circonstancié qui assume le fait qu'en contexte plura- 
liste, l'espace civique doit être pensé comme une construction sociale, de même que la solidarité nationale; que ni cet espace civique ni cette solidarité ne sont pour l'essentiel - gagés sur un substrat déjà là, ce qui confere à l'éducation scolaire un rôle crucial et très large (Conseil supérieur de l'éducation, 1998).

\section{En France, un changement amorcé, sans le mot interculturel}

Le renouvellement normatif s'est amorcé plus récemment en France; il est aussi politiquement plus difficile. En matière scolaire, il se heurte au cadre ordinaire de l'institution, qui ne reconnaît guère l'élève comme sujet de droits et offre peu d'espace à la participation. "L'école se veut le berceau de la citoyenneté, tout en restant à l'abri de toute pratique citoyenne», notent Barrère et Martuccelli (1998, p. 663). Elément symptomatique: le débat autour du défi que dresse l'islam devant la laïcité scolaire a été réglé juridiquement par le Conseil d'Etat, de façon libérale (la liberté est la règle, l'interdiction l'exception), mais les «affaires» de foulard demeurent sans solution légitime (les décisions judiciaires de réintégration des jeunes filles sont d'ordinaire récusées par le corps enseignant). Le renouvellement normatif se heurte aussi, dans l'espace public, aux réticences d'une mouvance intellectuelle encline à mobiliser pour la défense d'un acquis, sans s'attarder sur ses limites («L'universel républicain est un horizon indépassable», écrit Dominique Schnapper, 2000).

Les changements intervenus depuis 1998 au point de vue de la prise en compte du pluralisme n'en sont que plus notables. Les plus forts sont liés à la reconnaissance de l'existence des discriminations ethniques. Ainsi, la lutte contre les discriminations au travail et devant le travail a reçu une traduction législative qui met à niveau dans ce secteur la loi française avec la norme européenne, beaucoup plus protectrice que ne l'était la règle de droit antérieure: désormais la loi réprime non seulement la discrimination directe mais aussi la discrimination indirecte, elle élargit les possibilités de poursuites pénales et inverse la charge de la preuve devant les tribunaux prud'hommaux (loi du 16 novembre 2001).

Le domaine scolaire n'a pas connu une telle mutation, - même si l'on peut penser que le dispositif de lutte contre les discriminations modifie le contexte général de l'enseignement. La notion de discrimination indirecte n'a pas été étendue à l'école (pas plus qu'aux autres secteurs de l'action publique), bien que tout un chapitre du rapport 1998 du Haut conseil à l'intégration traite de faits qui semblent en relever. Quant à la thématique de la lutte contre les discriminations, elle n'a fait qu'une très discrète entrée dans le programme de l'ECJS de l'enseignement professionnel. L'éducation au non-racisme n'y figure pas. Néanmoins, la définition du programme et des méthodes de l'ECJS contient des ouvertures en direction d'une conception "délibérative pluraliste» de la citoyenneté. Le débat en classe - solidement documenté, exigent les instructions - en est l'instrument privilégié. La définition des thèmes, plus incitative que prescriptive, 
n'élude pas la question de la diversité (ethno)cuturelle, - même si celle-ci est présentée sous un angle général plutôt restrictif.

Il convient de faire réfléchir les élèves aux contradictions éventuelles des «cultures particulières» et de la "culture commune», dans le but de faire évoluer leurs pratiques. $\mathrm{La}$ "culture commune» n'est pas questionnée et on n'invite pas à le faire, on affirme sa nature politique: «La culture commune assurée par la citoyenneté repose sur des valeurs communes, celles qui assurent l'égale dignité de tous les hommes», affirmation qui peut aussi bien s'interpréter selon l'idéologie nationale classique (légitimant subrepticement la culture majoritaire) que selon une philosophie moderne de la démocratie (selon laquelle la culture commune est constituée essentiellement des valeurs démocratiques). Il reste que les élèves sont invités à se documenter et à discuter sur ces questions: une certaine place est faite à la divergence, peut-être au conflit si l'enseignant laisse la dynamique se créer dans le groupe. C'est une chance de voir une pratique éducative de la diversité s'affirmer au sein de l'école, à côté sinon à la place des pratiques contestataires et transgressives dont les élèves sont devenus coutumiers dans les secteurs difficiles ${ }^{3}$.

\section{Notes}

1 Les développements qui suivent sont adaptés d'un ouvrage à paraître sur l'école et l'ethnicité (Lorcerie, 2003).

2 Cet imaginaire nouveau ne recouvre pas les autres, cependant. L'imaginaire ethnonational, en crise à l'échelle nationale, trouve une nouvelle vigueur à l'échelle européenne, dans un surcroît d'altérisation des anciens colonisés. Vis-à-vis de ceux-ci, «nous» sommes encore plus «européens» qu'autrefois.

3 Cette orientation a été outillée de longue date par les praticiens chercheurs qui se sont inscrits dans le courant pédagogique issu de Célestin Freinet ou dans la pédagogie institutionnelle, - sans reconnaissance administrative.

\section{Références bibliographiques}

Audigier, F. \& Lagelée, G. (1996). Education civique et initiation juridique dans les collèges. Paris: INRP.

Barrère, A. \& Martuccelli, D. (1998). La citoyenneté à l'école: vers la définition d'une problématique sociologique. Revue française de sociologie, XXXIX (4), 651-671.

Barthon, C. (1998). Espaces et ségrégations scolaires. L'exemple des enfants d'immigrés dans les collèges de l'Académie de Versailles. Thèse de doctorat en géographie, dir. Michelle Guillon, Université de Poitiers.

Berque, J. (1985). L'Immigration à l'école de la République. Rapport au ministre de l'Education nationale. Paris: La Documentation française.

Citron, S. (1989). Le Mythe national. L'histoire de France en question. Paris: Les Editions ouvrières/Etudes et documentaton internationales.

Colas, D. (2000). La citoyenneté au risque de la nationalité. In M. Sadoun (Ed.), La Démocratie en France, 2. Limites (pp. 116-222). Paris: Gallimard.

Conseil supérieur de l'éducation (CSE) (1998), Eduquer à la citoyenneté. Québec: Rapport annuel sur l'état et les besoins de l'éducation. 
Cuche, D. (1996). La Notion de culture dans les sciences sociales. Paris: La Découverte.

Debarbieux, E. (1996). La Violence en milieu scolaire, 1. Etat des lieux. Paris: ESF.

Debarbieux, E., Garnier, A., Montoya, Y. \& Tichit, L. (1999). La Violence en milieu scolaire, 2. Le désordre des choses. Paris: ESF.

Déloye, Y. (1994). Ecole et citoyenneté. Lindividualisme républicain de Jules Ferry à Vichy: controverses. Paris: Presses de Sciences Po.

De Rudder, V., Poiret, Ch. \& Vourc'h, F. (2000). L’inégalité raciste. L'universalité républicaine à l'épreuve. Paris: PUF.

Elias, N. \& Scotson J.L. (1964/1997). The Established and the Outsiders. A sociological inquiry into community problems. trad. Logiques de l'exclusion, Enquête sociologique au coeur des problèmes d'une communauté. Avant-propos de M. Wieviorka. Paris: Fayard.

Habermas, J. (1996/1998). L'Intégration républicaine. Essais de théorie politique [titre or. Die Einbeziehung des Anderen, L'inclusion de l'autre]. Paris: Fayard.

Haut conseil à l'intégration (HCI) (1998). Lutte contre les discriminations: Faire respecter le principe d'égalité. Paris: La Documentation française.

Lorcerie, F. (1989). L'universalisme en cause? Les équivoques d'une circulaire sur la scolarisation des enfants d'immigrés. Mots, 18, 38-56.

Lorcerie, F. (1996). Laïcité 1996. La République à l'école de l'immigration? Revue française de pédagogie, 117, 53-85.

Lorcerie, F. (2000). La lutte contre les discriminations, ou l'intégration requalifiée. . VEI Enjeux, 121, 69-83.

Lorcerie, F. (Ed.). (2003). Le Défi de l'ethnicité (titre provisoire). Paris: Ed. ESF, [sous presse].

Mc Andrew, M. (2001). Immigration et diversité à l'école. Le débat québécois dans une perspective comparative. Montréal: Les Presses de l'Université de Montréal.

Mc Andrew, M. (2002). Immigration, pluralisme et éducation. In A.G. Gagnon (Ed.), Québec: Etat et société. 2ème édition. Montréal: Québec/Amérique.

Mauss, M. (1969 - écrit vers 1920). La nation. Oeuvres, vol. 3 (pp. 572-639). Paris: Ed. de Minuit.

Mesure, S. \& Renaut, A. (1999). Alter Ego. Les Paradoxes de l'identité démocratique. Paris: Aubier.

Noiriel, G. (1988). Le Creuset français. Histoire de l'immigration XIXe-XXe siècle. Paris: Seuil.

Nora, P. (1997). Lavisse, instituteur national, Les Lieux de mémoire. I, La Républiqu, (pp. 239275). Paris: Gallimard, coll. Quarto.

Ouellet, F. (1991). L'Education interculturelle. Essai sur le contenu de la formation des maîtres. Paris: L'Harmattan.

Ouellet, F. \& Pagé, M. (Ed.) (1991). Pluriethnicité, éducation et société. Construire un espace commun. Québec: IQRC.

Pagé, M. (2001). Léducation à la citoyenneté devant la diversité des conceptions de la citoyenneté. In M. Pagé, F. Ouellet \& L. Cortesao (Ed.), L'éducation à la citoyenneté (pp. 41 54). Sherbrooke: Ed. du CRP, Université de Sherbrooke.

Payet, J.P. (1995). Collèges de banlieue. Ethnographie d'un monde scolaire. Paris: MéridiensKlincsieck.

Payet, J.P. (1997). L'école et la construction de la citoyenneté. In A. van Zanten (Eds.), La Scolarisation dans les milieux "difficiles «. Politiques, processus, pratiques (pp. 99-113). Paris, INRP.

Porcher, L. (1981). L'Education des enfants de travailleurs migrants en Europe: L'interculturalisme et la formation des enseignants. Strasbourg: Conseil de l'Europe.

Rawls, J. (1993/1995). Libéralisme politique. Paris: PUF.

Sayad, A. (1988). L'école à l'épreuve de l'immigration. Vous avez dit ... Interculturel?, 3, 131-141.

Schnapper, D. (1991). La France de l'intégration. Sociologie de la nation en 1990. Paris: Gallimard.

Schnapper, D. (2000). L’universel républicain revisité. VEI Enjeux, 121, 10-22. 
Smith, A. (1991). National Identity. Reno, Las Vegas, London: University of Nevada Press.

Stora, B. (1999). Le Transfert d'une mémoire. De l'»Algérie française " au racisme anti-arabe. Paris: La Découverte.

Taylor, M. (1992). La politique de reconnaissance. In Ch. Taylor et al. (Eds.), Multiculturalisme (pp. 41-99). Paris: Aubier.

Tenzer, N. (1994). Philosophie politique. Paris: PUF.

Touraine, A. (1997). Pourrons-nous vivre ensemble? Egaux et différents. Paris: Fayard.

Van Zanten, A. (2001). L'Ecole de la périphérie. Paris: PUF.

Walzer, M. (1997/1998). On Toleration. Trad. Traité sur la tolérance. Paris: Gallimard.

Weber, M. (1956/1995). Les relations communautaires ethniques. Economie et société, vol. 2. Paris: Plon.

Weinstock, D. (1998). La citoyenneté comme «réponse « au problème du pluralisme des sociétés modernes. In J. Black, H. P. Glenn, D. Juteau \& D. Weinstock (Eds.), Les Enjeux de la citoyenneté. Un bilan interdisciplinaire (pp. 73-95). Montréal: Immigration et métropoles.

Wieviorka, M. (Ed.).(1996). Une société fragmentée? Le multiculturalisme en débat. Paris: La Découverte.

\section{Interkulturelle Erziehung und politische Bildung: Ein Irrweg der Soziologie der Nation}

\section{Zusammenfassung}

Die Frage nach dem Verhältnis zwischen interkultureller Erziehung und politischer Bildung wird zunehmend wieder in den soziopolitischen Kontext gestellt, in dem sie heute in westlichen Staaten gesehen werden muss. Dieser Kontext ist durch eine Krise bzw. - seltener - durch eine Ablehnung des ,klassischen' Nationalstaates gekennzeichnet, der im Hinblick auf die Einbürgerung auf der ethnischen Unterscheidung zwischen Etablierten und Aussenseitern, kombiniert mit einem demokratischen Prinzip, beruhte. Man kann das neue soziale Hervortreten der kulturellen Pluralität als Problem, das nach einer öffentlichen Bearbeitung ruft, und des Pluralismus als Wert beobachten. Die öffentliche Bildung ist einer der Orte dieser Krise, gleichzeitig einer der Orte, an denen deren Bearbeitung in Betracht gezogen wird. So wurde, in Québec, die Interkulturalität zum Emblem im Rahmen der symbolischen Wiederherstellung der québec'schen Nation (seit Ende der 1970er-Jahre), bevor die politische Macht stärker inklusive Embleme in den Vordergrund rückte. In Frankreich erklären sich die jüngste Revitalisierung der politischen Bildung und die (bescheidene) Anerkennung der kulturellen Differenzen ebenfalls im Kontext dessen, was man die Krise der assimilationsorientierten Integration nennen könnte, und der Wende hin zu einem «Regime der Toleranz» der Differenzen. 


\title{
Educazione interculturale e educazione alla cittadinanza: una lettura nell'ottica della sociologia della nazione
}

Riassunto

E' utile ricollocare la questione del rapporto tra educazione multiculturale e educazione alla cittadinanza nel suo contesto attuale e cioè quello sociopolitico dei paesi occidentali. Ciò che caratterizza questo contesto è una crisi, più raramente anche un rifiuto dello Stato-nazione «classico» che si fondava su un'opposizione etnica naturalizzata tra established e outsiders in combinazione con un principio democratico. Si osserva una nuova apparizione sociale della pluralità culturale quale problema che richiama un intervento pubblico e del pluralismo come valore. L'educazione pubblica è nel contempo luogo dove questa crisi si rivela e dove può essere affrontata. Ecco perché nel Québec l'emblema dell'interculturalità è stato sperimentato a partire dalla fine degli anni ' 70 nel contesto della rielaborazione simbolica della nazione prima che il potere politico lo eclissasse a beneficio di altri emblemi più esplicitamente integrativi. In Francia la recente riattivazione dell'educazione alla cittadinanza e il timido riconoscimento delle differenze culturali si spiega pure nel contesto di ciò che si può definire nei termini di una crisi dell'integrazione assimilazionista e della flessione del «regime di tolleranza» delle differenze.

\section{Intercultual education and citizen education: A detour by way of sociology of the nation}

\begin{abstract}
The question of the relationship between intercultural education and citizen education could be placed within a socio-political context or should be asked today in the western world. This context is characterized by a rarely seen crisis, namely the rejection of the "classical» nation state placed upon a ethnic naturalized opposition which lies somewhere between established and outsiders combined with a democratic principle. One observes a new social salience of cultural plurality as a problem, which is pleading for public treatment, and pluralism as a value. Public education is one of the origins of this crisis and at the same time is one of the places in which its treatment is foreseen. In Quebec, the emblem of interculturalism, they could experiment in the domain of the symbolic re-elaboration of Quebec as a nation (since the end of the 1970s) before being shut down by public opinion in favor of other emblems, which were more explicitly inclusive. In France the recent revitalization of citizen education and the recognition of different cultures explains the differences seen within the context of what we call the crisis of assimilated integration and the inflexion which sheds light on the «regime of tolerance».
\end{abstract}


T h e m a 